hep-th/0009127, UTPT-00-11

\title{
The Cosmological Constant and Warped Extra Dimensions
}

\author{
Hael Collins ${ }^{\dagger}$ and Bob Holdom ${ }^{\ddagger}$ \\ Department of Physics \\ University of Toronto \\ Toronto, Ontario M5S 1A7, Canada
}

\begin{abstract}
We study the behavior of a general gravitational action, including quadratic terms in the curvature, supplemented by a compact scalar field in $4+1$ dimensions. The generalized Einstein equation for this system admits solutions which are compact in one direction and Poincaré invariant in the remaining directions. These solutions do not require any fine-tuning of the parameters in the action - including the cosmological constant - only that they should satisfy some mild inequalities. Some of these inequalities can be expressed in a universal form that does not depend on the number of extra compact dimensions when the scenario is generalized beyond $4+1$ dimensions.
\end{abstract}

September, 2000

$\dagger$ hael@physics.utoronto.ca

$\ddagger$ bob.holdomQutoronto.ca 


\section{Introduction.}

The old idea that the universe might contain more than the observed four space-time dimensions has re-emerged recently in novel attempts to explain the weakness of gravity compared to the other forces [1] and the hierarchy problem [2], but it was realized earlier [3] that such theories might be able to address the cosmological constant problem [4]. The hope is that with extra dimensions, the metric might be able, through a non-trivial dependence on the extra coordinates, both to accommodate an arbitrary value for the cosmological constant and to maintain Poincaré invariance in $3+1$ of the directions. In their original treatment, Rubakov and Shaposhnikov [3] found that this idea could be realized with two extra dimensions but that the bulk metric contained a singularity. Moreover, their system was shown to be unstable by [5]. The more recent scenarios [2] involving 3-branes reintroduce the cosmological constant problem in the form of a fine-tuning between the bulk cosmological constant and the brane tension. The attempts by [6] and [7] to avoid this fine-tuning again resulted in naked singularities in the bulk [8].

We pursue the original idea of Rubakov and Shaposhnikov - that a non-factorizable metric that depends on an extra dimension could account for the presence of a cosmological constant. In particular, we seek metrics that are periodic in this extra dimension so that it can be made naturally compact when the period of the metric is identified with the compactification radius.

The standard Einstein equation for a theory without any 3-branes but with a cosmological constant does not permit metrics that simultaneously satisfy both requirements - that the metric is periodic and free of singularities. We therefore consider the effect of including higher order terms in the gravitational action which contain two powers of the curvature tensor [9], such as a Gauss-Bonnet term [10] [11] [12]. Such terms can be regarded as the next natural terms in an effective theory of the gravitational action such as might arise in the low-energy expansion of some quantum theory of gravity.

We show that a general five-dimensional gravitational action including all terms up to fourth order in derivatives when supplemented by a compact scalar field permits metrics that are periodic in the fifth coordinate and preserve Poincaré invariance in the other four dimensions. This result does not require any fine-tuning at the level of the parameters in the action - including the cosmological constant - other than that they should satisfy some mild bounds. Moreover, since these solutions are smooth and contain no singularities, either in the metric or in the scalar field, the scenario does not require any 3-branes with an accompanying requisite fine-tuning between the brane tension and bulk cosmological constant. The only requirement is that the compactification radius should be sufficiently small so as not to produce any discrepancies with current experiments.

In the next section, we review the solution to Einstein's equations in a $5 d$ theory with a cosmological constant and a free scalar field. Section three derives the field equations from a general action that includes quadratic terms in the curvature. In section four, we present several exact solutions of these field equations and further show that with the Gauss-Bonnet term alone the metric does not have periodic, smooth, non-singular solutions with $4 d$ Poincare invariance. The fifth section shows the requirements that a general $R^{2}$ action should satisfy to admit periodic, smooth, non-singular metrics and discusses a few representative cases found numerically. Section six examines a few general properties of 
including higher order terms in the effective action and discusses theories with more than one extra dimension. Section seven concludes.

\section{Background.}

In order to show the importance of higher derivative terms in the action, we first examine the solutions for a five-dimensional theory with only the standard Einstein-Hilbert action and a free scalar field, 1

$$
S=M_{5}^{3} \int d^{4} x d y \sqrt{-g}(2 \Lambda+R)-\frac{1}{2} \int d^{4} x d y \sqrt{-g}\left(\nabla_{a} \phi \nabla^{a} \phi\right)
$$

Here $\Lambda$ and $M_{5}$ are respectively the cosmological constant and the 5 dimensional Planck constant. $g_{a b}$ is the metric for the space-time. We denote the coordinates that correspond to the usual space-time dimensions by $x^{\mu}$, where $\mu, \nu, \cdots=0,1,2,3$, and the fifth coordinate by $y$, with $a, b, c, \ldots=0,1,2,3, y$. Ignoring the scalar field, when $\Lambda>0$ the universe is an anti-de Sitter (AdS) space-time while $\Lambda<0$ corresponds to a de Sitter (dS) space-time. We shall often work in units in which $M_{5}=1$.

When the metric has the form

$$
d s^{2}=g_{a b} d x^{a} d x^{b}=e^{A(y)} \eta_{\mu \nu} d x^{\mu} d x^{\nu}+d y^{2},
$$

the $\mu \mu$ and $y y$ components of the Einstein equation for (2.1) are

$$
\begin{aligned}
-\frac{3}{2}\left(A^{\prime}\right)^{2}-\frac{3}{2} A^{\prime \prime} & =-\Lambda+\frac{1}{4}\left(\phi^{\prime}\right)^{2} \\
-\frac{3}{2}\left(A^{\prime}\right)^{2} & =-\Lambda-\frac{1}{4}\left(\phi^{\prime}\right)^{2}
\end{aligned}
$$

while the equation for the scalar field is

$$
\phi^{\prime \prime}+2 A^{\prime} \phi^{\prime}=0
$$

A solution to equations $(2.3)-(2.4)$ is given by

$$
\begin{aligned}
& e^{A(y)}=e^{A_{0}}\left[\cos \left(2 \sqrt{-\frac{2}{3} \Lambda}\left(y-y_{0}\right)\right)\right]^{1 / 2}, \\
& \phi^{\prime}(y)=2 \sqrt{-\Lambda} \sec \left(2 \sqrt{-\frac{2}{3} \Lambda}\left(y-y_{0}\right)\right)
\end{aligned}
$$

where $A_{0}$ and $y_{0}$ are constants of integration, in addition to the trivial $\phi^{\prime}(y)=0$ solution. When $\Lambda<0$ (dS), we are able to obtain periodic solutions. However, the scalar field periodically becomes singular and the metric becomes imaginary. In order to remove this

1 Our convention for the signature of the metric is $(-,+,+,+,+)$ while the Riemann curvature tensor is defined by $-R_{b c d}^{a} \equiv \partial_{d} \Gamma_{b c}^{a}-\partial_{c} \Gamma_{b d}^{a}+\Gamma_{e d}^{a} \Gamma_{b c}^{e}-\Gamma_{e c}^{a} \Gamma_{b d}^{e}$. 
unacceptable behavior from the space-time, we require 3-branes to cut-off the manifold in extra dimension before the ill-behaved region is encountered. When $\Lambda>0(\operatorname{AdS})$, only the $\phi^{\prime}(y)=0$ solution is real and the theory can only be compact if we return to the original Randall-Sundrum scenario [2]. Thus for either sign of $\Lambda$, the scenario must contain some 3-branes which necessitates a fine-tuning of the brane tension with the value of the cosmological constant.

In the following, we shall see how the addition of a general $R^{2}$ action can lead to an acceptable dependence of the warp function, $A(y)$, on an extra compact dimension. Note that such an action encounters a difficulty when the extra dimension is not compact [13], as noted in [14]. If we consider variations about a flat $4 d$ metric, $\eta_{\mu \nu} \rightarrow g_{\mu \nu}^{(4)}\left(x^{\lambda}\right)$, we can relate the five-dimensional curvature for (2.2) to the four-dimensional curvature through

$$
R\left(x^{\mu}, y\right)=e^{-A(y)} R^{(4)}\left(x^{\mu}\right)+\cdots,
$$

where $R^{(4)}$ is the curvature associated with $g_{\mu \nu}^{(4)}$. By integrating out the extra dimension,

$$
\int d^{4} x d y \sqrt{g} M_{5}^{3} R\left(x^{\mu}, y\right)=\int d^{4} x \sqrt{g^{(4)}} M_{5}^{3} R^{(4)}\left(x^{\mu}\right) \int d y e^{A(y)}+\cdots,
$$

we determine the effective Planck's constant $M_{4}$ measured by a four-dimensional observer in terms of the $5 d$ Planck's constant $M_{5}$ through $M_{4}^{2} \equiv M_{5}^{3} \int d y e^{A(y)}$. As long as this integral is finite we can define a four dimensional effective theory of gravity. This argument fails at the next order since

$$
\int d^{4} x d y \sqrt{g} R^{2}=\int d^{4} x \sqrt{g^{(4)}}\left(R^{(4)}\right)^{2} \int d y 1+\cdots,
$$

so that in an effective theory, this term receives a correction proportional to the volume of the extra dimension. The problem only worsens at higher orders where the $\left[R^{(4)}\right]^{k}$ terms receive an enhancement of $\int d y e^{(2-k) A(y)}$. One way [14] to evade this difficulty occurs when the term induced in the $4 d$ effective theory is purely topological, as is the case for the $4 d$ Gauss-Bonnet term. In this paper we instead consider a scenario with compact extra dimensions along with a general $R^{2}$ action; a Gauss-Bonnet term alone is insufficient for our picture, as we show below.

\section{Gravity from a Generalized Action.}

A generic action with up to four derivatives of metric can be written as

$$
S=S_{\phi}+M_{5}^{3} \int d^{4} x d y \sqrt{-g}\left(2 \Lambda+R+a R^{2}+b R_{a b} R^{a b}+c R_{a b c d} R^{a b c d}+\cdots\right) .
$$

The additional terms can be interpreted as a squared Weyl tensor,

$$
C_{a b c d} C^{a b c d}=\frac{1}{6} R^{2}-\frac{4}{3} R_{a b} R^{a b}+R_{a b c d} R^{a b c d}
$$


a Gauss-Bonnet term,

$$
E=R^{2}-4 R_{a b} R^{a b}+R_{a b c d} R^{a b c d},
$$

and a third possible independent term,

$$
\mathcal{T}=\frac{1}{2} R^{2}-3 R_{a b} R^{a b}+R_{a b c d} R^{a b c d}
$$

The Weyl term vanishes when the metric is conformally flat, as is the case for (2.2), so we are free to add some multiple of the Weyl term to (3.4) without affecting the field equations. Later we shall use $\mu$ and $\lambda$ to denote the coefficients of the third term (3.4) and Gauss-Bonnet term respectively:

$$
\mu \equiv 16 a+5 b+4 c \quad 3 \lambda \equiv 10 a+2 b+c .
$$

We include the effect of a scalar field, initially through the action for a free field,

$$
S_{\phi}=\int d^{4} x d y \sqrt{-g}\left(-\frac{1}{2} k_{0} \nabla_{a} \phi \nabla^{a} \phi\right)
$$

although later we shall add a term $\left(\nabla_{a} \phi \nabla^{a} \phi\right)^{2}$ which is of the same order as the $R^{2}$ terms. Note that we have included a coefficient $k_{0}$ in equation (3.6).

Upon varying the action with respect to the metric we obtain [15]

$$
\begin{aligned}
& -g_{a b} \Lambda+R_{a b}-\frac{1}{2} g_{a b} R-\frac{1}{2} g_{a b}\left[a R^{2}+b R_{c d} R^{c d}+R_{c d e f} R^{c d e f}\right] \\
& \quad+2 a R R_{a b}-4 c R_{a c} R_{b}^{c}+2 c R_{a c d f} R_{b}^{c d f}-2(b+2 c) R^{c d} R_{a c d b} \\
& +\frac{1}{2}(4 a+b) g_{a b} \nabla^{2} R-(2 a+b+2 c) \nabla_{a} \nabla_{b} R+(b+4 c) \nabla^{2} R_{a b}=T_{a b}
\end{aligned}
$$

where $T_{a b}$ represents the energy-momentum tensor for the scalar field,

$$
T_{a b} \equiv \frac{1}{2} k_{0}\left[\nabla_{a} \phi \nabla_{b} \phi-\frac{1}{2} g_{a b} \nabla_{c} \phi \nabla^{c} \phi\right]
$$

For a non-factorizable metric of the form of equation (2.2), the sum of the $\mu \mu$ and the $y y$ components of (3.7) yields an equation in which the free scalar field does not appear,

$$
\begin{aligned}
& \mu\left[\frac{1}{2} A^{\prime \prime \prime \prime}+3 A^{\prime} A^{\prime \prime \prime}+\left(A^{\prime \prime}\right)^{2}+4 A^{\prime \prime}\left(A^{\prime}\right)^{2}\right] \\
& \quad+\frac{3}{2} \lambda\left[A^{\prime \prime}\left(A^{\prime}\right)^{2}+\left(A^{\prime}\right)^{4}\right]-3\left(A^{\prime}\right)^{2}-\frac{3}{2} A^{\prime \prime}=-2 \Lambda,
\end{aligned}
$$

so that the difference of these components can be used to determine its behavior,

$$
k_{0}\left(\phi^{\prime}\right)^{2}=\mu\left[A^{\prime \prime \prime \prime}+2 A^{\prime} A^{\prime \prime \prime}+4\left(A^{\prime \prime}\right)^{2}\right]+3 \lambda A^{\prime \prime}\left(A^{\prime}\right)^{2}-3 A^{\prime \prime} .
$$

The scalar field equation appears in (2.4), but it is not independent being a consequence of (3.9) and (3.10). When $A(y)$ is a periodic function of $y$, then (3.10) implies that $\phi^{\prime}(y)$ should also be periodic. Since this equation is non-linear in $A(y)$, the integral of $\phi^{\prime}(y)$ over one period is in general finite and non-zero so $\phi$ must itself be compact. Any additional dependence of the action on $\phi$, rather than on its derivatives, must be through periodic functions. 


\section{Analytical Solutions.}

The full fourth-order set of differential equations for a theory with a free scalar field and a set of $R^{2}$ terms with arbitrary coefficients does not admit a simple analytic solution except for special cases. In this section, we study two such examples of exact solutions. Although these solutions do not produce warp functions $A(y)$ that are smooth, non-singular and periodic, they provide partial boundaries for the region of the $\{\Lambda, \lambda, \mu\}$ parameter space in which we have found such solutions numerically. One of these surfaces requires both the $\mu$ and $\lambda$ terms in the field equations. Among this class of solutions is a metric which falls off exponentially as $y \rightarrow \pm \infty$, as in [13] but without the need of a 3-brane. A second of the surfaces, $\mu=0$, represents a theory with only a Gauss-Bonnet term at the $R^{2}$ order. Note that a third boundary lies along the surface $\Lambda=0$ where we can trivially satisfy the field equations (3.9), (3.10) and (2.4) with constant solutions, $A(y)=A_{0}$ and $\phi(y)=\phi_{0}$. We have found non-trivial periodic solutions only when $\Lambda<0$ and $\mu<0$.

\subsection{An Exact Solution.}

We can discover an interesting set of exact solutions by noting that the linear combination of field equations that eliminates the scalar field, (3.9), does not depend on $A(y)$ except through its derivatives and does not contain the fifth coordinate explicitly. Together these properties allow (3.9) to be recast as a second order differential equation,

$$
\begin{aligned}
\mu\left[\frac{1}{2} P^{2} \frac{d^{2} P}{d z^{2}}+\frac{1}{2} P\left(\frac{d P}{d z}\right)^{2}\right. & \left.+3 z P \frac{d P}{d z}+P^{2}+4 P z^{2}\right] \\
+ & +\frac{3}{2} \lambda\left[P z^{2}+z^{4}\right]-3 z^{2}-\frac{3}{2} P=-2 \Lambda,
\end{aligned}
$$

through the introduction of

$$
z \equiv \frac{d A}{d y} \quad \text { and } \quad P(z) \equiv \frac{d z}{d y} .
$$

A simple set of solutions in the full $\{\Lambda, \lambda, \mu\}$ parameter space is found by substituting

$$
P(z)=a z^{2}+b
$$

into (4.1) which imposes the constraints

$$
\Lambda=\frac{3}{4} \frac{b}{a+2} \quad \lambda=-\frac{a}{b} \frac{3 a+4}{a+2} \quad \mu=\frac{3}{2} \frac{1}{b} \frac{1}{a+2} .
$$

The resulting warp function $A(y)$ is periodic provided that $a b>0$ :

$$
e^{A(y)}=e^{A_{0}}\left[\cos \left(\sqrt{a b}\left(y-y_{0}\right)\right)\right]^{-1 / a} .
$$

Note that this solution for $A(y)$, when substituted into (3.10), implies that the scalar field is constant, $\phi^{\prime}(y)=0$. 
In general, this solution is not satisfactory since it contains singularities when $a>0$ and becomes complex for generic values of $a<0$. When $a=-1 / 2 n$, where $n$ is a positive integer, the behavior improves so that $e^{A(y)}$ is everywhere real and non-singular when $\Lambda<0$, although for this case $e^{A(y)}$ vanishes at regular intervals. We shall find in the next section that the set of solutions in (4.5) forms a boundary in the $\{\Lambda, \lambda, \mu\}$ parameter space beyond which periodic, non-vanishing solutions exist when $\Lambda<0$ and $\mu<0$.

We can also use (4.5) to generate a variant of the usual Randall-Sundrum scenario [13], but without including a 3-brane. For example, when $a>0$ and $\Lambda<0$, we find that

$$
e^{A(y)}=e^{A_{0}} \operatorname{sech}\left(\sqrt{-\frac{4}{3} a(a+2) \Lambda}\left(y-y_{0}\right)\right)^{1 / a} .
$$

Although this solution requires fine-tuning the parameters in the action, as given in (4.4), it achieves a metric whose zero mode is centered about $y=y_{0}$ and which falls off exponentially as $y \rightarrow \pm \infty$. Unlike previous examples [16] which used a thick brane to obtain this behavior, (4.6) does not require any scalar field. Since this theory has an infinite extra dimension, some method of trapping the Standard Model fields near $y=y_{0}$ is further required.

The endpoints of the set of solutions in (4.6), shown in figure 1, have interesting properties. Taking $a \rightarrow \infty\left(\mu=0, \lambda \Lambda=-\frac{9}{4}\right)$, so that the region in which the first derivative of the warp function smoothly changes sign has a vanishing thickness, the solution (4.6) approaches the warp function studied by Randall and Sundrum [13,

$$
e^{A(y)}=e^{A_{0}} e^{-2 \sqrt{\frac{-\Lambda}{3}}\left|y-y_{0}\right|} .
$$

At the other endpoint, $a \rightarrow 0\left(\lambda=0, \mu \Lambda=\frac{9}{32}\right)$, the warp function produces a Gaussian metric

$$
e^{A(y)}=e^{A_{0}} e^{\frac{4 \Lambda}{3}\left(y-y_{0}\right)^{2}}
$$

which for $\Lambda<0$ decreases more rapidly away from $y=y_{0}$ than the standard RandallSundrum metric.

\subsection{Insufficiency of a Gauss-Bonnet Term Alone.}

A theory with only a Gauss-Bonnet contribution to the $R^{2}$ action has the advantage of producing a set of differential equations with no more than second derivatives of the warp function $A(y)$. Unfortunately, for our purpose restricting to such a theory too greatly constrains the form of the solutions. Without the $\mu$-terms, equation (3.9) implies that all the extrema of $A(y)$, if they exist, are all local maxima or all local minima depending upon the sign of $\Lambda$ since at the points where $A^{\prime}(y)=0, A^{\prime \prime}(y)=\frac{4}{3} \Lambda$. However, the surface $\mu=0$ is still important as it provides a partial boundary to the region in the full $\{\Lambda, \lambda, \mu\}$ parameter space in which satisfactory, periodic solutions do exist.

When the metric has the form (2.2), any warp function $A(y)$ that has an extremum will encounter a singularity only a finite distance away from it. Integrating the scalar field 


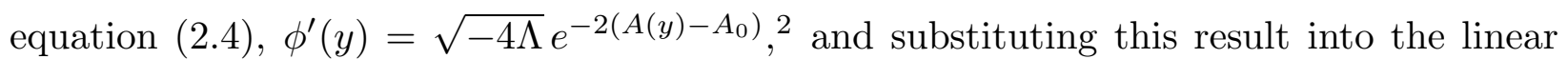
combination of (3.9) and (3.10) that eliminates $A^{\prime \prime}(y)$ when $\mu=0$ yields a first order differential equation. Its solution determines $A(y)$ implicitly:

$$
\pm\left(y-y_{0}\right)=\frac{\sqrt{\lambda}}{2 \sqrt{x_{0}+1}} \operatorname{arctanh}\left(\sqrt{\frac{1 \pm x}{x_{0}+1}}\right)-\frac{\sqrt{\lambda}}{2 \sqrt{x_{0}-1}} \arctan \left(\sqrt{\frac{1 \pm x}{x_{0}-1}}\right)
$$

where

$$
x \equiv \sqrt{x_{0}^{2}+\frac{4}{3} \lambda \Lambda e^{-4\left(A(y)-A_{0}\right)}} \quad \text { and } \quad x_{0} \equiv \sqrt{1-\frac{4}{3} \lambda \Lambda} .
$$

The signs on the right side of (4.10) cannot be chosen separately; however, the minus sign should be used to obtain $A^{\prime}(y)=0$ at some point. In the limit $\lambda \rightarrow 0$, (4.10) reproduces $(2.5)$.

In the interesting $\Lambda<0$ region, from the expression (4.10) we observe that no acceptable solutions exist for any value of $\lambda$. For example when $\lambda<0, A(y)$ becomes singular in (4.10) at a finite distance from $y_{0}$. For $\lambda>0$, the warp function also contains a singularity, but this time it appears in its second derivative. Solving equation (3.9) for $A^{\prime \prime}(y)$, we note that it becomes singular whenever $A^{\prime}(y)= \pm \lambda^{-1 / 2}$, which again occurs at a finite value of $y$. Appendix A contains a fuller discussion of the properties of the solution (4.10) and the location of its singularities.

\section{An Analysis of the Full $R^{2}$ Action.}

Before describing our approach for finding numerical solutions and presenting several representative examples, we summarize with a sketch of the parameter space in figure 1. The unshaded area of the figure, which lies in the $\Lambda<0$ and $\mu<0$ region of the $\{\Lambda, \lambda, \mu\}$ parameter space, shows where smooth, non-singular, periodic warp functions exist. We can describe another boundary by solving for $\lambda$ as a function of $\mu$ in (4.4),

$$
\lambda \Lambda=-\frac{8}{3} \mu \Lambda \pm 4 \sqrt{2 \mu \Lambda}-\frac{9}{4}
$$

we immediately observe that the shape of the curve of solutions (4.5) is the same for all values of the cosmological constant, $\Lambda<0$, if the $\lambda$ and $\mu$ axes are appropriately rescaled. This feature corresponds to a rescaling invariance of the field equation (3.9) under $y \rightarrow \sigma y$,

2 With $\phi^{\prime}(y)=0$, the only possible solutions are those of the form,

$$
A(y)= \pm\left[1 \pm \sqrt{1-\frac{4}{3} \lambda \Lambda}\right]^{1 / 2} \frac{y-y_{0}}{\sqrt{\lambda}}+A_{0}
$$

where any of the four possible sign choices is allowed. The case of a scalar field in a $\Lambda=0$ theory was solved in [11]. 
$\mu \rightarrow \sigma^{2} \mu, \lambda \rightarrow \sigma^{2} \lambda$ and $\Lambda \rightarrow \sigma^{-2} \Lambda$, where $\sigma$ is a real constant. Therefore, we can express our results in terms of two dimensionless parameters, $\{\lambda \Lambda, \mu \Lambda\}$.

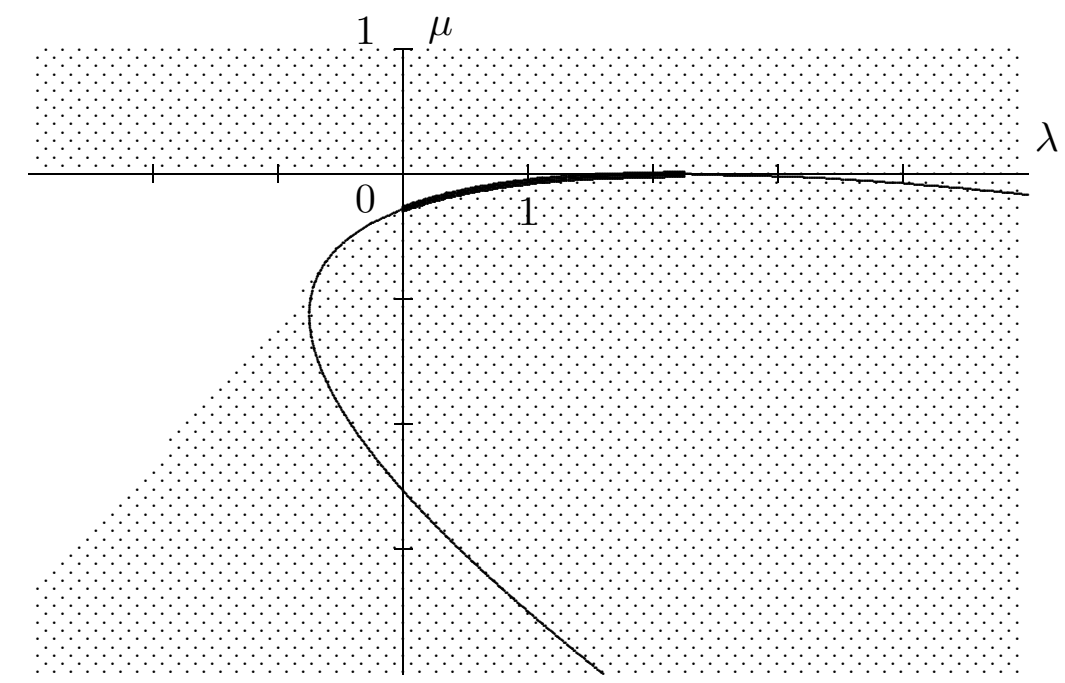

Figure 1. A plot of the parameter space $\{\lambda, \mu\}$. For convenience, we have chosen $\Lambda=-1$ in generating this figure. The shaded region does not appear to contain periodic solutions for $A(y)$ while in the unshaded region, we have found periodic solutions numerically for arbitrarily chosen points. The curve depicts the surface of solutions of equation (4.5); the darker line shows the location of the solutions in (4.6).

A periodic solution for a generic set of values of $\Lambda, \lambda$ and $\mu$ is found by numerically integrating the differential equation (3.9). The coordinate $y$ does not explicitly appear in the equation (3.9) which moreover only depends on the warp function through its derivatives. Thus, we can always translate by $y \rightarrow y-y_{0}$ and $A(y) \rightarrow A(y)+A_{0}$ to obtain another solution. Therefore we can choose $A(0)=A^{\prime}(0)=0$ without any loss of generality. We also chose $A^{\prime \prime \prime}(0)=0$ which limits our solutions to those that are even about the origin, although we did not find any periodic solutions that are odd when we relaxed this constraint. The subsequent evolution of the warp function away from $y=0$ then depended solely upon the initial value of the second derivative, $A^{\prime \prime}(0)$.

An arbitrary value for $A^{\prime \prime}(0)$, given some set of values for $\{\lambda \Lambda, \mu \Lambda\}$, does not lead to a periodic solution. Generically, the warp function tended to reach a singularity at a finite distance or to approach asymptotically a solution of the form $A^{\prime}(y)=$ constant as $y \rightarrow \infty$. Between these two extremes, precisely chosen values of $A^{\prime \prime}(0)$ produce periodic solutions for arbitrarily chosen values of $\lambda \Lambda$ and $\mu \Lambda$ within the unshaded region of figure 1 . This result demonstrates the existence of periodic solutions which naturally select a compactification radius without the need to add a 3-brane to the theory or to fine-tune the parameters in the action (3.1), as long as they lie within the allowed region of parameter space.

When the scalar field enters the action only through a free kinetic term, the value of $k_{0}\left(\phi^{\prime}\right)^{2}$ is negative for all the periodic solutions that we found numerically. Although this result implies that the kinetic energy term has the wrong sign, it appears to be an artifact of truncating the scalar action; this unphysical feature disappears when higher order terms appear in the scalar action. An example of such a solution is placed at the end of this section. 
One region of parameter space that admits a semi-analytic approximation of the solution is where $\Lambda \mu \sim \epsilon^{2} \ll 1$ with $\Lambda \lambda \gg 1$. In this region we can try an oscillating solution for the warp function of the form

$$
A(y)=A_{0}+\epsilon \cos \left(\omega\left(y-y_{0}\right)\right)+A_{2} \epsilon^{2} \cos \left(2 \omega\left(y-y_{0}\right)\right)+\mathcal{O}\left(\epsilon^{3}\right)
$$

for a small amplitude, $\epsilon$. When substituted into (3.9), the only terms that are $\mathcal{O}(\epsilon)$ are those linear in $A(y)$ :

$$
\frac{1}{2} \mu A^{\prime \prime \prime \prime}-\frac{3}{2} A^{\prime \prime}=\mathcal{O}\left(\epsilon^{2}\right)
$$

which, to leading order, requires $\omega^{2} \approx-3 / \mu$ so that the period of the compact dimension is

$$
y_{c} \approx 2 \pi \sqrt{-\mu / 3} .
$$

The $\mathcal{O}\left(\epsilon^{2}\right)$ terms, which arise from the following terms in (3.9),

$$
\frac{1}{2} \mu A^{\prime \prime \prime \prime}+3 \mu A^{\prime} A^{\prime \prime \prime}+\mu\left(A^{\prime \prime}\right)^{2}-3\left(A^{\prime}\right)^{2}-\frac{3}{2} A^{\prime \prime}=-2 \Lambda+\mathcal{O}\left(\epsilon^{3}\right),
$$

determine the value of $\epsilon$ in terms of the cosmological constant,

$$
\epsilon^{2} \approx-\frac{4}{3} \frac{\Lambda}{\omega^{2}}
$$

and the coefficient of the $\mathcal{O}\left(\epsilon^{2}\right)$ term in the warp function: $A_{2}=-\frac{1}{4}$. Thus, for the linear terms to dominate requires that $\Lambda \mu \approx \frac{9}{4} \epsilon^{2} \ll 1$. Note that since the $\lambda$ terms in the field equations contain at least three powers of the warp function, to this order $\lambda$ can be arbitrary provided it does not alter the $\epsilon$-expansion: $\Lambda \lambda \ngtr 1$.

This semi-analytic estimate is confirmed when we plot the numerical solution to (3.9) for $\Lambda=-1, \lambda=0$ and $\mu=-0.01$ shown in figure 2. Holding $\Lambda=-1$ and $\lambda=0$ fixed and varying $\mu$, we have checked that the period and amplitude of the numerical solutions is better and better approximated by (5.4) and (5.6) as we let $\mu \rightarrow 0$.

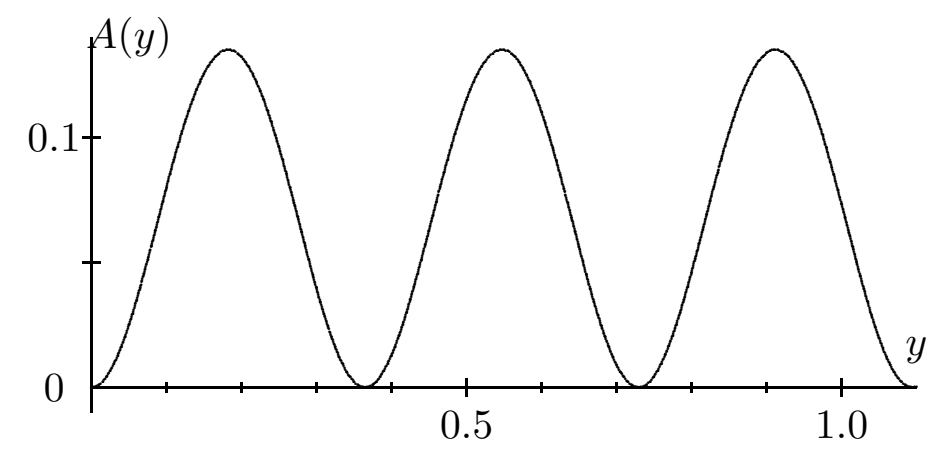

Figure 2. A periodic warp function $A(y)$ for $\Lambda=-1, \lambda=0$ and $\mu=-0.01$. The initial condition is $A^{\prime \prime}(0)=21.460889$. The value of $k_{0}\left(\phi^{\prime}\right)^{2}$ oscillates about -4 , also with a small period and amplitude.

We can find numerical solutions arbitrarily close to the surface $\mu=0$, for $\lambda<\frac{9}{4}$ and $\Lambda=-1$, and to the surface described by (5.1), provided $\mu>-\frac{81}{128}$. Apparently, another 
boundary exists for solutions with $\mu<-\frac{81}{128}$ which extends approximately along the line $\mu \sim \frac{9}{8} \lambda$. We have indicated this boundary in figure 1 by extending the shaded region beyond the curve (5.1). As $\lambda$ and $\mu$ approach this boundary from above, the numerical solutions grow more cusped as in the more extreme example depicted in figure 3 where the warp function has broad maxima and sharp, deep minima. The amplitude is also significantly larger so that $e^{A(y)}$ changes by several orders of magnitude. Although the figure appears to have cusps at the minima, the function $A(y)$ is actually smooth at these points and has no discontinuities in the slope.

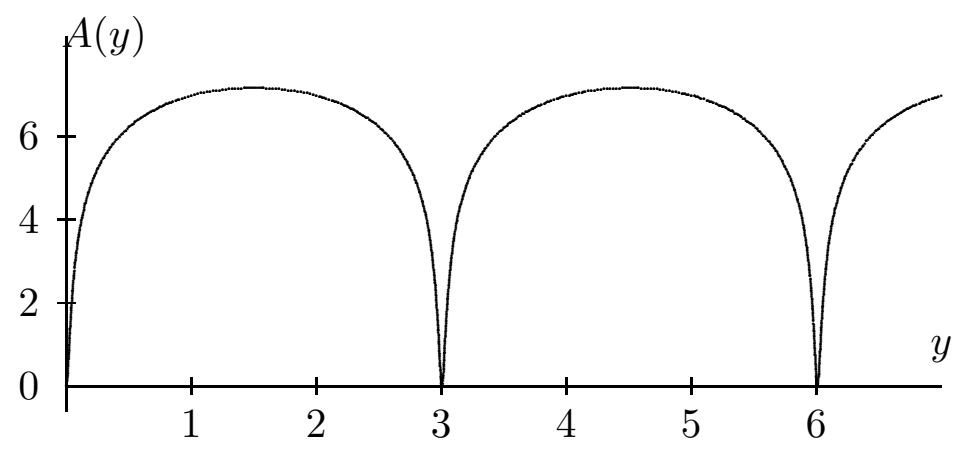

Figure 3. A periodic warp function $A(y)$ for $\Lambda=\lambda=\mu=-1$. The initial condition is $A^{\prime \prime}(0)=5367.89$. Despite their cusped appearance, the minima are smooth.

Most solutions within the unshaded region of figure 1 tend to lie between the extremes depicted in figures 2 and 3 as in the example provided by the following sketch. More examples are presented in Appendix B.

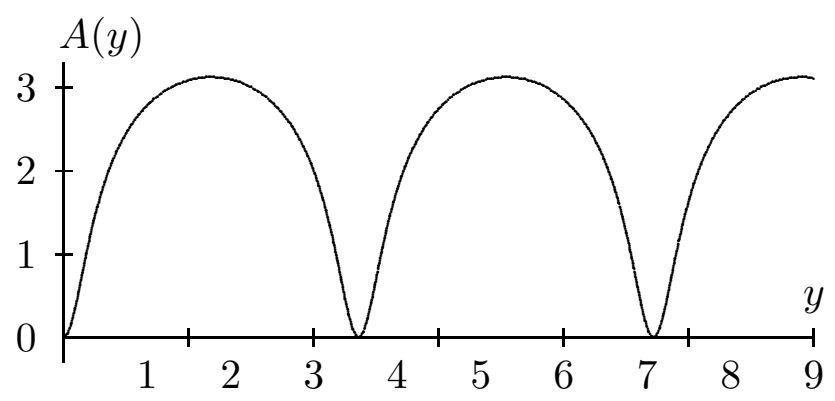

Figure 4. An example of a periodic warp function that lies between the extremes represented by figures 2 and 3 . For this sketch we used $\Lambda=-1, \lambda=-3$ and $\mu=-2$. The initial condition is $A^{\prime \prime}(0)=42.2832125$.

Finally, we must address whether the kinetic energy term in the scalar action can have the correct sign, $k_{0}=1$, when $A(y)$ is periodic. If we include the additional term 3

$$
-\frac{1}{4} k_{1}\left(\nabla_{a} \phi \nabla^{a} \phi\right)^{2}
$$

3 Other terms of the same order are possible, such as $R_{a b} \nabla^{a} \phi \nabla^{b} \phi$, but the squared kinetic term is the simplest to analyze. 
then the field equations (3.7) require

$$
\begin{aligned}
\mu\left[\frac{1}{2} A^{\prime \prime \prime \prime}+2 A^{\prime} A^{\prime \prime \prime}+\right. & \left.\frac{3}{2}\left(A^{\prime \prime}\right)^{2}+2 A^{\prime \prime}\left(A^{\prime}\right)^{2}\right] \\
+\frac{3}{4} \lambda\left[2 A^{\prime \prime}\left(A^{\prime}\right)^{2}+\left(A^{\prime}\right)^{4}\right]-\frac{3}{2}\left(A^{\prime}\right)^{2}-\frac{3}{2} A^{\prime \prime} & =-\Lambda+\frac{1}{4} k_{0}\left(\phi^{\prime}\right)^{2}+\frac{1}{8} k_{1}\left(\phi^{\prime}\right)^{4} \\
\mu\left[A^{\prime} A^{\prime \prime \prime}-\frac{1}{2}\left(A^{\prime \prime}\right)^{2}+2 A^{\prime \prime}\left(A^{\prime}\right)^{2}\right] & \\
+\frac{3}{4} \lambda\left(A^{\prime}\right)^{4}-\frac{3}{2}\left(A^{\prime}\right)^{2} & =-\Lambda-\frac{1}{4} k_{0}\left(\phi^{\prime}\right)^{2}-\frac{3}{8} k_{1}\left(\phi^{\prime}\right)^{4} .
\end{aligned}
$$

Since these expressions are only quadratic in $\left(\phi^{\prime}(y)\right)^{2}$, we can solve for

$$
\begin{aligned}
\left(\phi^{\prime}(y)\right)^{2}=\frac{k_{0}}{3 k_{1}}[-1 \pm & \left\{1-24 \frac{k_{1}}{k_{0}^{2}}\left[\Lambda+\mu\left(A^{\prime} A^{\prime \prime \prime}-\frac{1}{2}\left(A^{\prime \prime}\right)^{2}+2 A^{\prime \prime}\left(A^{\prime}\right)^{2}\right)\right.\right. \\
& \left.\left.\left.+\frac{3}{4} \lambda\left(A^{\prime}\right)^{4}-\frac{3}{2}\left(A^{\prime}\right)^{2}\right]\right\}^{1 / 2}\right]
\end{aligned}
$$

and substitute the result into (5.8) to obtain a differential equation for $A(y)$. This equation still admits periodic solutions such as the example sketched in figure 5 for $\Lambda=-1, \lambda=0$, $\mu=-0.1, k_{0}=1$, and $k_{1}=-0.25$ and choosing the minus root $\operatorname{tin}$ (5.9). The value of $k_{0}\left(\phi^{\prime}\right)^{2}$ is positive so we obtain the standard normalization for the scalar kinetic energy.

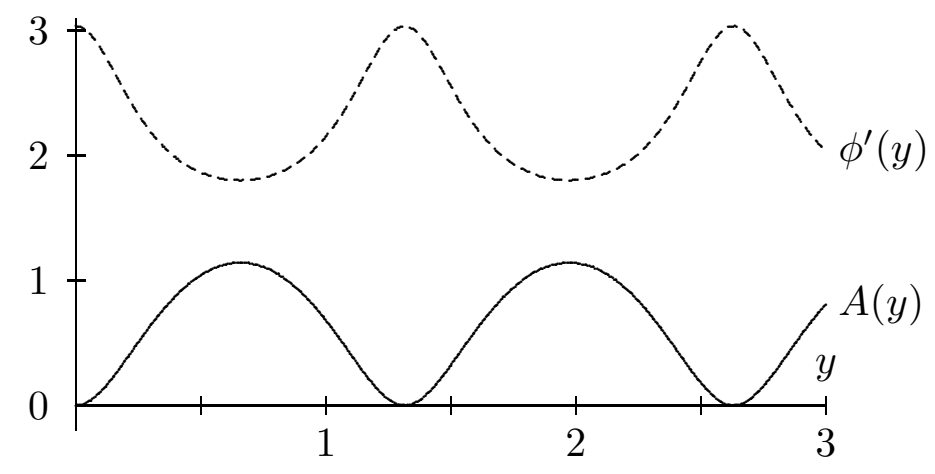

Figure 5. A periodic warp function $A(y)$ (solid line) and $\phi^{\prime}(y)$ (dashed line) for $\Lambda=-1, \lambda=0$, $\mu=-0.1, k_{0}=1$, and $k_{1}=-0.25$. The initial condition is $A^{\prime \prime}(0)=23.77364592$.

\section{Discussion.}

\subsection{Higher Order Terms in the Effective Action.}

The gravitational action that we have considered should be regarded as only the first few terms of a possibly infinite effective action arranged in powers of derivatives. Therefore, we might worry whether higher order terms will spoil the periodic behavior seen in the last section. Here we briefly motivate why the existence of smooth, periodic, non-singular solutions might be a generic feature of this scenario.

4 The choice of the plus root gives a periodic solution that again requires $k_{0}<0$. 
The small $\Lambda$ region of the generalized parameter space, formed by the coefficients of the terms in the effective action, still should admit warp functions that approximate sinusoids as in (5.2),

$$
A(y)=A_{0}+\epsilon \cos \left(\omega\left(y-y_{0}\right)\right)+\mathcal{O}\left(\epsilon^{2}\right) .
$$

For this approximate solution, the amplitude of the oscillations is assumed to be small, $\mathcal{O}(\epsilon)$, so terms in the equations of motion with fewer powers of $A(y)$ tend to dominate the shape of the warp function. For an action with a general set of $R^{k}$ terms included, the leading behavior in the $\epsilon \ll 1$ limit is

$$
-\frac{3}{2} \frac{d^{2} A}{d y^{2}}+\frac{1}{2} \mu \frac{d^{4} A}{d y^{4}}+\sum_{k=3}^{\infty} \sum_{i} \mu_{k, i} \frac{d^{2 k} A}{d y^{2 k}}=\mathcal{O}\left(\epsilon^{2}\right)
$$

where the $\mu_{k, i}$ are some linear combinations of the coefficients of the $R^{k}$ terms. When the warp function is of the form (6.1), these linear terms have solutions as long as the coefficients $\mu_{k, i}$ are such that the equation

$$
\frac{3}{2}+\frac{1}{2} \mu \omega^{2}+\sum_{k=3}^{\infty} \sum_{i}(-1)^{k} \mu_{k, i} \omega^{2(k-1)}=0
$$

has a real root. As before, the cosmological constant can be related to the amplitude $\epsilon$, by solving for the $\mathcal{O}\left(\epsilon^{2}\right)$ terms of the field equations of the full Lagrangian. Thus, when the cosmological constant is small, $\Lambda \sim \mathcal{O}\left(\epsilon^{2}\right)$, then the full parameter space contains a region in which the warp function is, up to $\mathcal{O}\left(\epsilon^{2}\right)$ corrections, given by a sinusoid (6.1). As with the parameter $\lambda$ in the $R^{2}$ action, the other parameters which appear in the full equations of motion, which we denote by $\lambda_{k, i}$, only appear in terms that are at least cubic in powers of the warp function and so only need to satisfy the weaker requirement that they do not ruin the $\epsilon$ expansion, $\lambda_{k, i} \Lambda \gg 1$.

The problem of establishing the existence of more general periodic solutions becomes only more difficult at higher orders. However, well-behaved periodic solutions do seem generically to exist for subsets of the terms of the general field equations other than the linear terms. As an example, one such subset of the terms in (3.9),

$$
\frac{1}{2} \mu A^{\prime \prime \prime \prime}+\left[4 \mu+\frac{3}{2} \lambda\right] A^{\prime \prime}\left(A^{\prime}\right)^{2}=0
$$

also has periodic solutions when $\frac{\lambda}{\mu}>-\frac{8}{3}$. One subset of the terms in the $R^{3}$ action is

$$
A^{\prime \prime \prime \prime \prime \prime \prime}+c_{1} A^{\prime \prime \prime \prime}\left(A^{\prime}\right)^{2}-c_{2} A^{\prime \prime}\left(A^{\prime}\right)^{4}=0,
$$

which has periodic solutions for some subregion of the space $c_{1}, c_{2}>0$. This behavior also suggests that it is plausible that periodic solutions should exist in some region of the enlarged parameter space when higher order terms are included in the gravitational action. 


\subsection{The Scenario in d Dimensions.}

Although for clarity we have concentrated on a scenario in which the universe contains $3+1$ infinite dimensions and one extra compact dimension, the picture can be generalized to higher dimensions with many of the features found above intact. The most trivial modification of the metric (2.2) would be of the form

$$
d s^{2}=e^{A(y)}\left[\eta_{\mu \nu} d x^{\mu} d x^{\nu}+\sum_{i=1}^{d-5} d z_{i}^{2}\right]+d y^{2}
$$

for a theory in $d$ total space-time dimensions. Although the warp function $A(y)$ must still be a periodic function of $y$, the $z_{i}$ directions can be trivially compactified with arbitrary compactification radii. The generalized Einstein equation (3.7) for this scenario produces the following two independent differential equations,

$$
\begin{aligned}
\tilde{\mu}\left[\frac{1}{2} A^{\prime \prime \prime \prime}+\frac{d-1}{2} A^{\prime} A^{\prime \prime \prime}+\right. & \left.\frac{3(d-1)}{8}\left(A^{\prime \prime}\right)^{2}+\frac{(d-1)^{2}}{8} A^{\prime \prime}\left(A^{\prime}\right)^{2}\right] \\
+\tilde{\lambda}\left[A^{\prime \prime}\left(A^{\prime}\right)^{2}+\frac{d-1}{8}\left(A^{\prime}\right)^{4}\right]-\frac{(d-1)(d-2)}{8}\left(A^{\prime}\right)^{2}-\frac{d-2}{2} A^{\prime \prime} & =-\Lambda+\frac{1}{4} k_{0}\left(\phi^{\prime}\right)^{2} \\
\frac{d-1}{4} \tilde{\mu}\left[A^{\prime} A^{\prime \prime \prime}-\frac{1}{2}\left(A^{\prime \prime}\right)^{2}+\frac{d-1}{2} A^{\prime \prime}\left(A^{\prime}\right)^{2}\right] & \\
+\frac{d-1}{8} \tilde{\lambda}\left(A^{\prime}\right)^{4}-\frac{(d-1)(d-2)}{8}\left(A^{\prime}\right)^{2} & =-\Lambda-\frac{1}{4} k_{0}\left(\phi^{\prime}\right)^{2} .
\end{aligned}
$$

As before, only two of the three possible linear combinations of $R^{2}$ terms contribute,

$$
\tilde{\mu}=4(d-1) a+d b+4 c \quad \tilde{\lambda}=\frac{d-4}{4}[d(d-1) a+(d-1) b+2 c] .
$$

As in the $d=5$ case, for a conformally flat metric such as (6.6) the squared Weyl term $C_{a b c d} C^{a b c d}$ does not contribute to the equations of motion (6.7). $\tilde{\lambda}$ vanishes for $d=4$ since the Gauss-Bonnet term then corresponds to the Euler form and is a topological density. Incidentally, for the solution to be periodic in this unphysical case which only has $2+1$ dimensional Poincaré invariance, $\mu$ must be moderately fine-tuned to lie within the interval $-\frac{1}{24}<\mu<0$.

An interesting feature of this more general field equation is that the analytic solutions of (4.5) are still solutions when $d>5$. The generalization of (4.4) is then

$$
\begin{aligned}
\Lambda & \equiv \frac{(d-1)(d-2)^{2}}{36} \bar{\Lambda}=\frac{b}{8} \frac{(d-1)(d-2)}{2 a+d-1} \\
\tilde{\lambda} & \equiv \frac{3}{2} \bar{\lambda}=-\frac{a}{b} \frac{(d-2)(3 a+d-1)}{2 a+d-1} \\
\tilde{\mu} & \equiv \frac{16 \bar{\mu}}{(d-1)^{2}}=\frac{d-2}{b(2 a+d-1)} .
\end{aligned}
$$

5 Note that in (3.5) we have defined $\lambda=\left.\frac{2}{3} \tilde{\lambda}\right|_{d=5}$ to agree with the conventional normalization for the Gauss-Bonnet term. 
Here we have introduced a rescaled set of parameters, $\{\bar{\Lambda}, \bar{\lambda}, \bar{\mu}\}$, to emphasize that the shape of the curve shown in figure 1 (5.1) is in fact universal - it is the same for arbitrary $\Lambda<0$ and in arbitrary dimension $d>4$ when $\mu$ and $\lambda$ are appropriately rescaled:

$$
\bar{\lambda} \bar{\Lambda}=-\frac{8}{3} \bar{\mu} \bar{\Lambda} \pm 4 \sqrt{2 \bar{\mu} \bar{\Lambda}}-\frac{9}{4} .
$$

Note that while the location of this set of solutions is independent of $d$ and $\Lambda$ when plotted in the $\{\bar{\lambda} \bar{\Lambda}, \bar{\mu} \bar{\Lambda}\}$-plane, individual points along this curve do not correspond to the same solutions since both the period and the exponent in (4.5) depend on $d$.

\section{Conclusions.}

A theory with an extra compact dimension and an action with a set of $R^{2}$ terms and a compact scalar field contains sufficient freedom to permit a metric that maintains Poincaré invariance in $3+1$ of the dimensions without any fine-tuning of the terms in the action. Although the resulting field equations are fourth order, the existence of these metrics can be shown numerically. The examples that we have found are smooth, periodic and contain no singularities or zeros throughout space-time. As a check, we have confirmed that when the numerical solutions for the warp function and scalar field are substituted back into the action, the integral over the extra dimensions gives zero, so that the effective four dimensional cosmological constant vanishes. We have also found several exact solutions which, while they do not themselves provide satisfactory metrics, bound the region of parameter space in which exist the desired smooth, non-singular metrics that are periodic in the extra dimension. Moreover, these exact solutions continue to exist for an arbitrary number of extra dimensions and can be expressed in a universal, dimension-independent form.

As in the original proposal by Rubakov and Shaposhnikov [3] to address the cosmological constant problem using extra dimensions, the existence of metrics with periodic warp functions that are flat in the other $3+1$ dimensions leaves unanswered the question of whether they are preferred over other possibilities. For example, we have also found a class of metrics that, while still periodic in one dimension, correspond to $3+1$ de Sitter or anti-de Sitter spaces in the other components. There is also the question of stability, and whether these solutions can be reached from generic initial conditions. The answers to these problems are central to the search for a realistic cosmology, but the problems are dynamical in nature and a fine turning of fundamental parameters is now not obviously required.

\section{Acknowledgements}

H. C. has benefited from discussions with T. Chow. This work was supported in part by Natural Sciences and Engineering Research Council of Canada. 


\section{Appendix A. Singularities in the Solutions for a Gauss-Bonnet Action.}

In section 4.2, we mentioned that the warp function (4.10) for a Gauss-Bonnet action encounters singularities after only a finite interval in the extra dimension when $\Lambda<0$. Moreover, the character of these singularities depends upon the sign of $\lambda$. When $\lambda<0$, if we let $A(y) \rightarrow-\infty$ in (4.10), we discover that this divergence occurs at

$$
\pm\left(y-y_{0}\right)=\frac{\pi \sqrt{3}}{8} \frac{1}{\sqrt{-\Lambda}}\left[\sqrt{1+\sqrt{1-\frac{4}{3} \lambda \Lambda}}+\sqrt{1-\sqrt{1-\frac{4}{3} \lambda \Lambda}}\right] .
$$

Note that the sum on the right side is always real, provided $\lambda<0$ and $\Lambda<0$.

For $\lambda>0$, another type of singularity occurs since then it becomes possible to have $A^{\prime \prime}(y)$ diverge when $A^{\prime}(y)= \pm \lambda^{-1 / 2}$. (3.9) and (3.10) imply that $\sqrt{\lambda} A^{\prime}(y)= \pm \sqrt{1 \pm x}$, so inserting $x=0$ into (4.10), we see that these singularities also occur at a finite value of $y$,

$$
\pm\left(y-y_{0}\right)=+\frac{1}{2} \sqrt{\frac{\lambda}{x_{0}+1}} \operatorname{arctanh} \frac{1}{\sqrt{x_{0}+1}}-\frac{1}{2} \sqrt{\frac{\lambda}{x_{0}-1}} \arctan \frac{1}{\sqrt{x_{0}-1}} .
$$

Since $A^{\prime \prime}(y)=\frac{4}{3} \Lambda$ when $y$ is an extremum, when $\Lambda>0$ the warp function only contains minima. Thus we have the possibility that $A(y) \rightarrow+\infty$ which, from (4.10), occurs as $y \rightarrow \pm \infty$. However, for $\lambda \Lambda>\frac{3}{4}, A^{\prime \prime}(y)$ diverges at a finite value for $y$.

\section{Appendix B. Several More Examples.}

In the following table, we list the properties of a several more examples of periodic

\begin{tabular}{|c|c|c|c|c|}
\hline$\lambda$ & $\mu$ & $A^{\prime \prime}(0)$ & \multicolumn{2}{|c|}{ period $A_{\max }-A_{\min }$} \\
\hline$\frac{1}{10}$ & $-\frac{1}{10}$ & 8.38996422 & 1.2596 & 0.5174 \\
\hline 1 & $-\frac{1}{30}$ & 12.20618213 & 0.9608 & 0.4321 \\
\hline 0 & $-\frac{1}{4}$ & 8.96881784 & 2.4476 & 1.4186 \\
\hline 0 & $-\frac{1}{10}$ & 8.35597222 & 1.2264 & 0.4950 \\
\hline 0 & $-\frac{1}{1000}$ & 64.61423 & 0.1148 & 0.0422 \\
\hline-10 & -1 & 3.7145907 & 2.1898 & 0.6545 \\
\hline-1 & -1 & 5367.88 & 3.005 & 7.159 \\
\hline-10 & -10 & 34282 & 4.419 & 8.501 \\
\hline-256 & -256 & 10439 & 9.524 & 8.633 \\
\hline
\end{tabular}
solutions.

Table 1. A brief list of some of values of $\lambda$ and $\mu$ that give periodic, smooth, non-singular solutions when $\Lambda=-1$. 
In the table 2 , we hold $\lambda$ and $\mu$ fixed while varying $\Lambda$.

\begin{tabular}{|c|c|c|c|}
\hline$\Lambda$ & $A^{\prime \prime}(0)$ & \multicolumn{2}{|c|}{ period $A_{\max }-A_{\min }$} \\
\hline-20 & 494520 & 0.7210 & 8.0777 \\
\hline-10 & 53680 & 0.9502 & 7.1593 \\
\hline-4 & 87404 & 1.9086 & 7.4489 \\
\hline-4 & 47.264364 & 1.4166 & 1.9249 \\
\hline-2 & 14.5276037 & 1.262 & 0.7918 \\
\hline$-\frac{1}{2}$ & 5.32842445 & 1.1708 & 0.3155 \\
\hline$-\frac{\overline{1}}{4}$ & 3.5543619 & 1.1586 & 0.2167 \\
\hline$-\frac{1}{10}$ & 2.14671387 & 1.1517 & 0.1348 \\
\hline
\end{tabular}

Table 2. A list of values of the cosmological constant $\Lambda$ that give periodic, smooth, non-singular solutions given $\lambda=\mu=-\frac{1}{10}$.

Note that a periodic solution for a specific choice of $\{\Lambda, \lambda, \mu\}$ is not necessarily unique; two different values for $A^{\prime \prime}(0)$ can lead to two different periodic solutions. An example of this phenomenon is listed in table 2 and illustrated in figure 5.

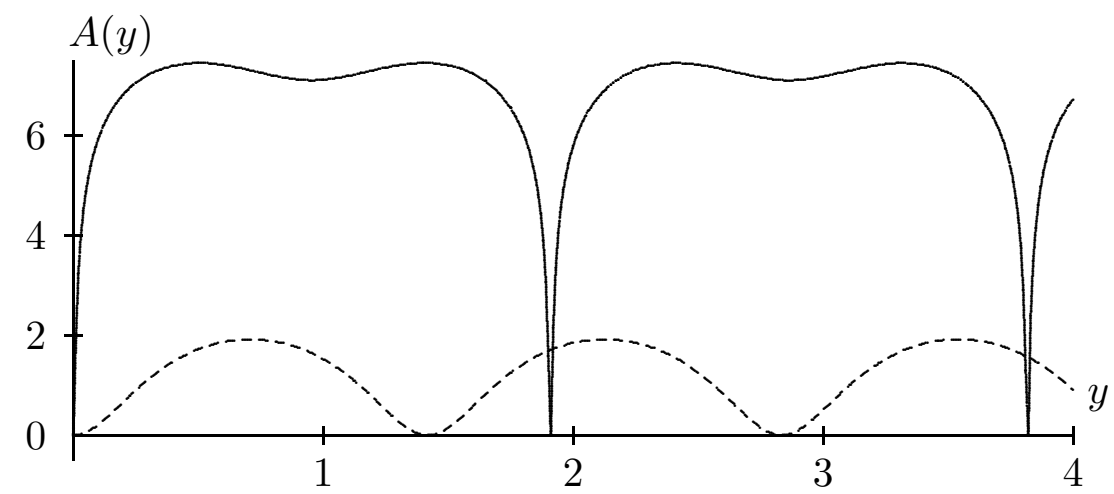

Figure 6. A pair of periodic warp function $A(y)$ for $\Lambda=-4$ and $\lambda=\mu=-0.1$. The initial condition for the solid curve is $A^{\prime \prime}(0)=87404$ while that of the dashed curve is $A^{\prime \prime}(0)=47.264364$. Again, both functions are smooth everywhere. 


\section{References}

[1] N. Arkani-Hamed, S. Dimopoulos and G. Dvali, "The hierarchy problem and new dimensions at a millimeter," Phys. Lett. B429, 263 (1998) hep-ph/9803315 and I. Antoniadis, N. Arkani-Hamed, S. Dimopoulos and G. Dvali, "New dimensions at a millimeter to a Fermi and superstrings at a TeV," Phys. Lett. B436, 257 (1998) hep-ph/9804398.

[2] L. Randall and R. Sundrum, "A large mass hierarchy from a small extra dimension," Phys. Rev. Lett. 83, 3370 (1999) hep-ph/9905221.

[3] V. A. Rubakov and M. E. Shaposhnikov, "Extra Space-Time Dimensions: Towards A Solution To The Cosmological Constant Problem," Phys. Lett. B125, 139 (1983).

[4] S. Weinberg, "The Cosmological Constant Problem," Rev. Mod. Phys. 61, 1 (1989).

[5] G. V. Lavrelashvili and P. G. Tinyakov, "On Possible Spontaneous Compactification Leading To Zero Cosmological Constant," Sov. J. Nucl. Phys. 41, 172 (1985).

[6] N. Arkani-Hamed, S. Dimopoulos, N. Kaloper and R. Sundrum, "A small cosmological constant from a large extra dimension," Phys. Lett. B480, 193 (2000) hepth/0001197].

[7] S. Kachru, M. Schulz and E. Silverstein, "Bounds on curved domain walls in 5d gravity," hep-th/0002121.

[8] See for example S. S. Gubser, "Curvature singularities: The good, the bad, and the naked," hep-th/0002160; C. Csaki, J. Erlich, C. Grojean and T. Hollowood, "General properties of the self-tuning domain wall approach to the cosmological constant problem," hep-th/0004133 or P. Binetruy, J. M. Cline and C. Grojean, "Dynamical instability of brane solutions with a self-tuning cosmological constant," hep-th/0007029.

[9] C. Wetterich, "Spontaneous Compactification In Higher Dimensional Gravity," Phys. Lett. B113, 377 (1982) and Q. Shafi and C. Wetterich, "Cosmology From Higher Dimensional Gravity," Phys. Lett. B129, 387 (1983).

[10] J. E. Kim, B. Kyae and H. M. Lee, "Effective Gauss-Bonnet interaction in RandallSundrum compactification," Phys. Rev. D62, 045013 (2000) hep-ph/9912344 and J. E. Kim, B. Kyae and H. M. Lee, "Various modified solutions of the RandallSundrum model with the Gauss-Bonnet interaction," Nucl. Phys. B582, 296 (2000) hep-th/0004005.

[11] I. Low and A. Zee, "Naked singularity and Gauss-Bonnet term in brane world scenarios," hep-th/0004124.

[12] N. Deruelle and T. Dolezel, "Brane versus shell cosmologies in Einstein and EinsteinGauss-Bonnet theories," gr-qc/0004021.

[13] L. Randall and R. Sundrum, "An alternative to compactification," Phys. Rev. Lett. 83, 4690 (1999) [hep-th/9906064. 
[14] Z. Kakushadze, "Localized (super)gravity and cosmological constant," hep-th/0005217 and "Gravity in the Randall-Sundrum brane world revisited," hep-th/0008128.

[15] B. S. DeWitt, "Dynamical Theory of Groups and Fields," New York: Gordon and Breach (1965).

[16] M. Gremm, "Four-dimensional gravity on a thick domain wall," Phys. Lett. B478, 434 (2000) hep-th/9912060. 